\title{
Use of the Intravascular Contrast Agent NC100150 Injection in Spin-Echo and Gradient-Echo Imaging of the Heart
}

\author{
Andrew M. Taylor, ${ }^{1}$ Jonathan R. Panting, ${ }^{1}$ Jennifer Keegan, ${ }^{1}$ \\ Peter D. Gatehouse, ${ }^{1}$ Permi Jhooti, ${ }^{1}$ Guang Z. Yang, ${ }^{1}$ \\ Steven McGill, ${ }^{2}$ Jane M. Francis, ${ }^{1}$ Elisabeth D. Burman, ${ }^{1}$ \\ David N. Firmin, ${ }^{1}$ and Dudley J. Pennell ${ }^{1}$ \\ ${ }^{1}$ Magnetic Resonance Unit, Royal Brompton Hospital, London, UK \\ ${ }^{2}$ Nycomed Imaging AS, Oslo, Norway, a part of Nycomed Amersham
}

\begin{abstract}
This is the first study of the intravascular iron oxide particle contrast agent, NC100150 Injection (Nycomed Imaging AS, Oslo, Norway, a part of Nycomed Amersham) in magnetic resonance imaging of the human heart. Eighteen healthy male volunteers were studied at both 0.5 and $1.5 \mathrm{~T}$ before and after the administration of NC100150 Injection. Transaxial spin-echo images were acquired at both field strengths, conventional gradient-echo cine images at $0.5 \mathrm{~T}$, and breathhold Turbo-FLASH cine images at 1.5 T. Optimized cine imaging sequences were used postcontrast, with a high flip angle of $60-70^{\circ}$. In the spin-echo images there was a significant reduction in the blood pool flow artifact at the level of the right atrium $(0.5 T, 57 \%, \mathrm{p}<0.01 ; 1.5 T, 41 \%, \mathrm{p}=0.01)$ and the left ventricle $(L V)$ $(0.5 \mathrm{~T}, 45 \%, \mathrm{p}=0.01 ; 1.5 \mathrm{~T}, 45 \%, \mathrm{p}<0.01)$. In the conventional gradient-echo cines at $0.5 \mathrm{~T}$, there was a significant increase in the LV blood pool and myocardial signal difference-to-noise ratio (SDNR) in the diastolic (56\%, $\mathrm{p}=0.01)$ and systolic $(141 \%, \mathrm{p}<0.001)$ frames. There was also a significant increase in the signal intensity (SI) gradient at the LV blood pool-myocardial border in the diastolic and systolic frames (both $\mathrm{p}<0.001$ ). At higher doses of NC100150 Injection ( 3 and $4 \mathrm{mg} / \mathrm{kg}$ ), a rim of signal void around the LV blood pool was observed, perfectly defining the LV blood poolmyocardial border. In the Turbo-FLASH breathhold cines at $1.5 \mathrm{~T}$, there was a significant increase in the LV blood pool-myocardial SDNR in the diastolic $(221 \%, \mathrm{p}<0.001)$ and systolic $(916 \%, \mathrm{p}<$ 0.001 ) frames. Again, there was also a significant increase in the SI gradient at the LV blood poolmyocardial border in the diastolic and systolic frames (both $\mathrm{p}=0.003$ ). In conclusion, NC100150 Injection was given safely to 18 healthy subjects. Image quality and LV blood pool-myocardial definition were improved after the administration of NC100150 Injection. These improvements enable better
\end{abstract}

Received February 12, 1998; Accepted May 14, 1998 Address reprint requests to Andrew M. Taylor. 
spin-echo anatomical definition, better definition of myocardial wall motion, and should improve the capability of automated edge detection algorithms.

KEY WORDS: Cardiac MR; Contrast agents; Intravascular; Iron-oxide particles.

\section{INTRODUCTION}

Dynamic magnetic resonance (MR) angiography signal-to-noise and image quality have been dramatically improved with the use of extracellular gadolinium-based contrast agents (1). The rapid clearance of extracellular contrast agents from the blood pool has limited their use in cardiac MR, where acquisition times and total scanning times remain relatively long. Furthermore, there is rapid myocardial uptake of extracellular gadolinium compounds, which limits the amount of blood pool-myocardial contrast seen with these agents in the heart (2).

MR imaging now represents the most accurate noninvasive method for measuring left ventricular (LV) volumes and mass $(3,4)$. Conventional gradient-echo imaging of the entire LV is time consuming, and breathhold techniques have been developed to reduce imaging times $(5,6)$. These techniques use short echo times (TE) and repeat times (TR), which reduce the acquisition time but also reduce signal to noise. Manual image analysis can also be time consuming, and attempts hăve been made to automate this process $(7,8)$. For accurate automated LV measurements, good blood pool-myocardial contrast is necessary. Thus, a contrast agent that increases LV blood pool signal while the myocardial signal remains largely unchanged, and remains in the blood pool for long periods of time, would be useful for gradient-echo imaging and automated image analysis.

Intravascular contrast agents can be divided into three groups: gadolinium chelates, manganese chelates, and iron oxide particles (9). In this study we report the phase I preliminary MR findings for spin-echo and gradientecho imaging of the heart, using for iron oxide particle contrast agent $\mathrm{NC1} 100150$ Injection (Nycomed Imaging AS, Oslo, Norway, a part of Nycomed Amersham).

\section{MATERIALS AND METHODS}

Eighteen healthy male subjects were studied at 0.5 and $1.5 \mathrm{~T}$ ( 9 subjects at each field strength, mean age $25 \mathrm{yr}$ ). Transaxial spin-echo images, from the level of the right pulmonary artery to the inferior border of the heart, and horizontal long-axis (HLA) gradient-echo cine images were acquired before and after the administration of $\mathrm{NC} 100150$ Injection. Three different doses of NC100150 Injection $(2,3$, and $4 \mathrm{mg} / \mathrm{kg}$ ) were given to three subjects at each field strength. All images were acquired with electrocardiogram gating. All subjects gave informed consent.

\section{Table 1}

Spin-Echo Noise Data ( $\pm S D$ ) for All Doses of NC100150 Injection at $0.5 T$ and $1.5 T$

\begin{tabular}{|c|c|c|c|c|c|c|}
\hline & \multicolumn{2}{|c|}{ Aorta } & \multicolumn{2}{|c|}{ RA } & \multicolumn{2}{|c|}{ LV } \\
\hline & Pre & Post & Pre & Post & Pre & Post \\
\hline \multicolumn{7}{|l|}{$0.5 \mathrm{~T}$} \\
\hline Noise & $97 \pm 51$ & $94 \pm 95$ & $147 \pm 113$ & $64 \pm 49$ & $80 \pm 56$ & $44 \pm 31$ \\
\hline$t$-test & \multicolumn{2}{|c|}{0.90} & \multicolumn{2}{|c|}{$<0.01$} & \multicolumn{2}{|c|}{0.01} \\
\hline$\%$ noise reduction & \multicolumn{2}{|c|}{4} & \multicolumn{2}{|c|}{57} & \multicolumn{2}{|c|}{45} \\
\hline \multicolumn{7}{|l|}{$1.5 \mathrm{~T}$} \\
\hline Noise & $40 \pm 19$ & $41 \pm 28$ & $78 \pm 34$ & $46 \pm 18$ & $119 \pm 40$ & $65 \pm 32$ \\
\hline$t$-test & \multicolumn{2}{|c|}{0.90} & \multicolumn{2}{|c|}{0.1} & \multicolumn{2}{|c|}{$<0.01$} \\
\hline$\%$ noise reduction & \multicolumn{2}{|c|}{ na } & \multicolumn{2}{|c|}{41} & \multicolumn{2}{|c|}{45} \\
\hline
\end{tabular}

Noise was measured within the aorta, right atrium (RA), and left ventricle (LV) before and after the administration of NC100105 Injection. The $t$-test and \% noise reduction compare data pre- and postcontrast at each level. na, not applicable. 


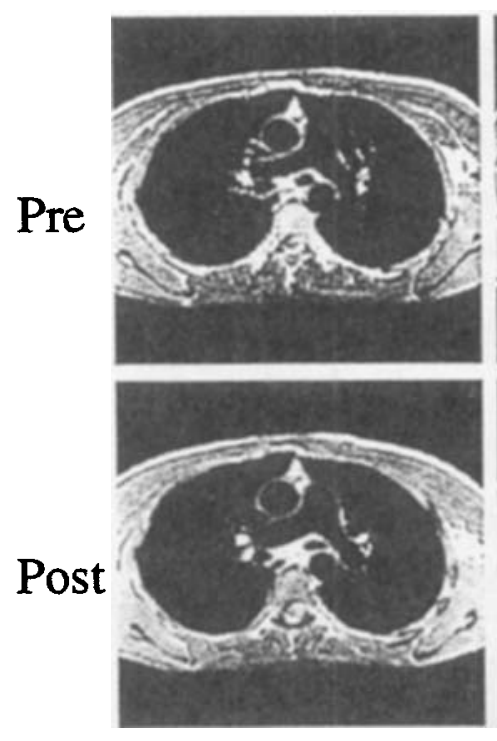

A.
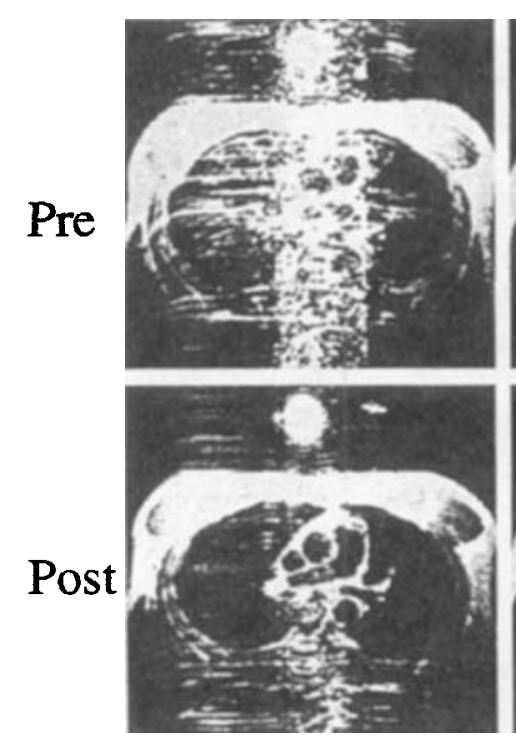

B.
Ao

Ao
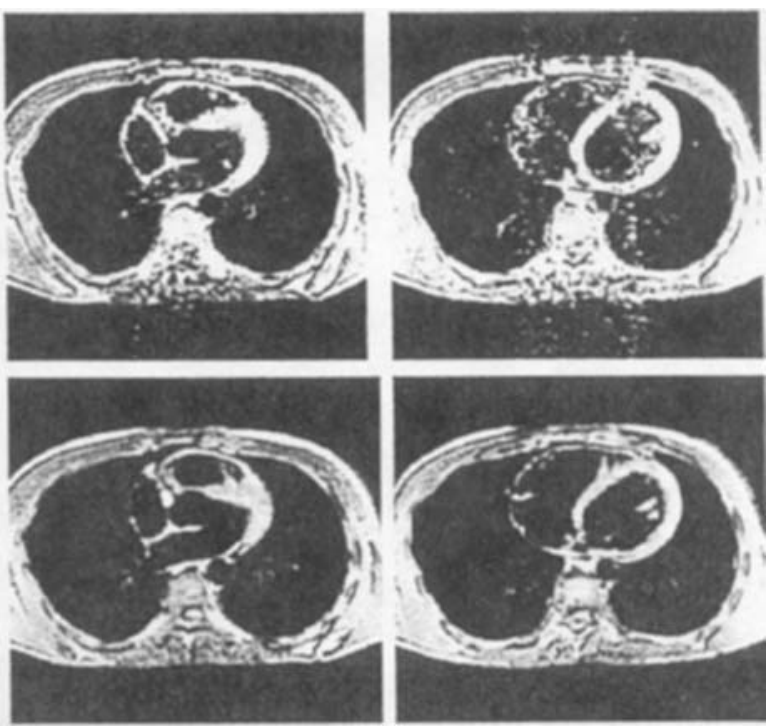

RA
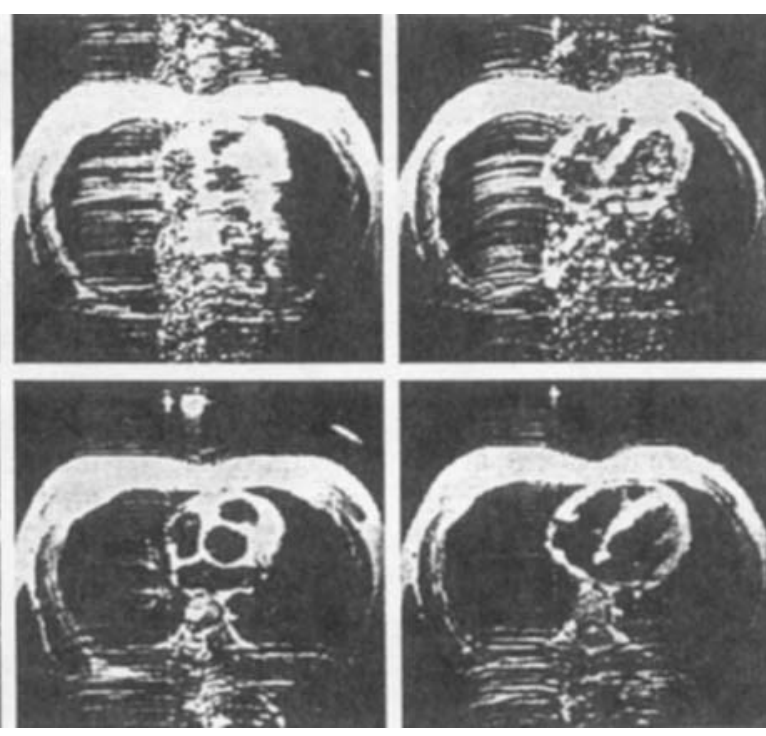

RA

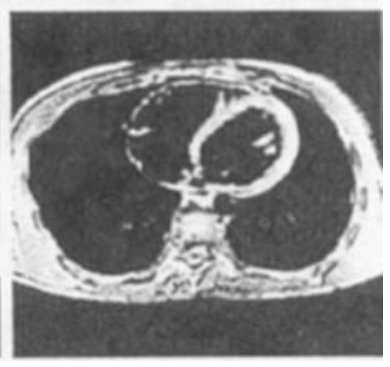

LV

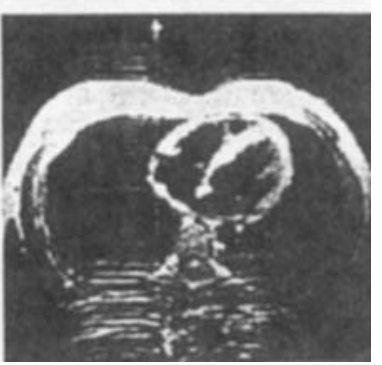

LV

Figure 1. Transaxial spin-echo images before and after the administration of NC100150 Injection. The image brightness and contrast settings are identical before and after contrast. Ao, level of aorta; RA, level of right atrium; LV, level of left ventricle. Image quality is improved by the reduction of the blood flow artifact. (A) Images acquired at $0.5 \mathrm{~T}(3 \mathrm{mg} / \mathrm{kg}$ ). (B) Images acquired at $1.5 \mathrm{~T}$ (4 $\mathrm{mg} / \mathrm{kg}$ ). Note in A that there is more respiratory artifact present in the precontrast than postcontrast images. 


\section{Imaging Protocol at $0.5 \mathrm{~T}$}

Imaging was performed on an inhouse-designed mobile $0.5-\mathrm{T}$ scanner, using a $20-\mathrm{cm}^{2}$ surface coil. An MR imaging console (Surrey Medical Imaging Systems, Surrey, UK) with additional hardware and software was used to generate and drive the gradient coils and radiofrequency waveforms and to receive and reconstruct the image data.

Spin-echo image parameters were as follows: TE 40 msec, TR, RR interval, slice thickness (SLT) $10 \mathrm{~mm}$, slices 10, field of view (FOV) $40 \mathrm{~cm}^{2}$, read matrix 256 , number of excitations (NEX) 2, phase matrix 128. Imaging parameters were identical after contrast. Mean postcontrast imaging time was $46 \mathrm{~min}$.

Conventional gradient-echo image parameters were as follows: TE $8 \mathrm{msec}$, TR $40 \mathrm{msec}$, SLT $10 \mathrm{~mm}$, FOV 40 $\mathrm{cm}^{2}$, read 256, phase 128 , NEX 2, frames 16 . The flip angle was $45^{\circ}$ before contrast and $70^{\circ}$ after contrast. Mean postcontrast imaging time was $38 \mathrm{~min}$.

\section{Imaging Protocol at $1.5 \mathrm{~T}$}

Imaging was performed on a Picker Edge 1.5-T scanner (Picker, Cleveland, $\mathrm{OH}$ ), using a phased array receiver coil. Spin-echo image parameters were as follows: TE $22 \mathrm{msec}$, TR RR interval, SLT $5 \mathrm{~mm}$, slice gap 5 mm, slices 10 , FOV $35 \mathrm{~cm}^{2}$, read 256, phase 128 , NEX 2. Imaging parameters were identical after contrast. Méan postcontrast imaging time was $54 \mathrm{~min}$.

A segmented gradient-echo Turbo-FLASH sequence, acquired during a single breathhold (méan acquisition time $15 \mathrm{sec}$ ), was used for the HLA cine images. Parameters were as follows: TE $2.7 \mathrm{msec}$, TR $6 \mathrm{msec}$, SLT 10 $\mathrm{mm}$, FOV $35 \mathrm{~cm}^{2}$, read 256 , phase 128 , views per frames 8 , NEX 1 , frames 12 . The flip angle was $30^{\circ}$ before contrast and 60 after contrast. Mean postcontrast imaging time was $37 \mathrm{~min}$.

\section{Image Analysis}

For the spin-echo images, blood pool noise was measured (signal SD) before and after the administration of $\mathrm{NC} 100150$ Injection in three slices in the ascending aorta (Ao), right atrium (RA), and LV, respectively. The image quality was also subjectively scored by two observers: 1 , very satisfactory; 2 , satisfactory; 3 , unsatisfactory; 4 , very unsatisfactory.

For the gradient echo images, a signal-to-noise ratio (SNR) was calculated for both the global LV blood pool and the LV myocardium, for the diastolic and systolic frames, before and after the administration of NC100150 Injection. Noise was measured as the SD of the signal in a region of interest (ROI) outside the phase encode artifact. The ROI was placed in an identical position before and after contrast. A signal difference- (global LV blood pool signal minus myocardial signal) to-noise ratio (SDNR) was also calculated for the diastolic and systolic frames before and after the administration of NC100150 Injection. The parameter SDNR was used because it provides a display-independent parameter that gives equivalent information to a contrast-to-noise ratio (10). The first frame of the cine was used as the diastolic frame. A measurement of the image signal intensity (SI) gradient was made by assessing the image profile across the LV blood pool-endocardial border in the midseptum position. The gradient of each image profile was determined for a linear fit after correction for background noise. Overall, cine quality and myocardial wall definition in the septum, apex, and lateral wall were subjectively scored, using the scoring system above.

Parametric data were compared with the Student's $t$-test. Nonparametric data were compared with the Wil-

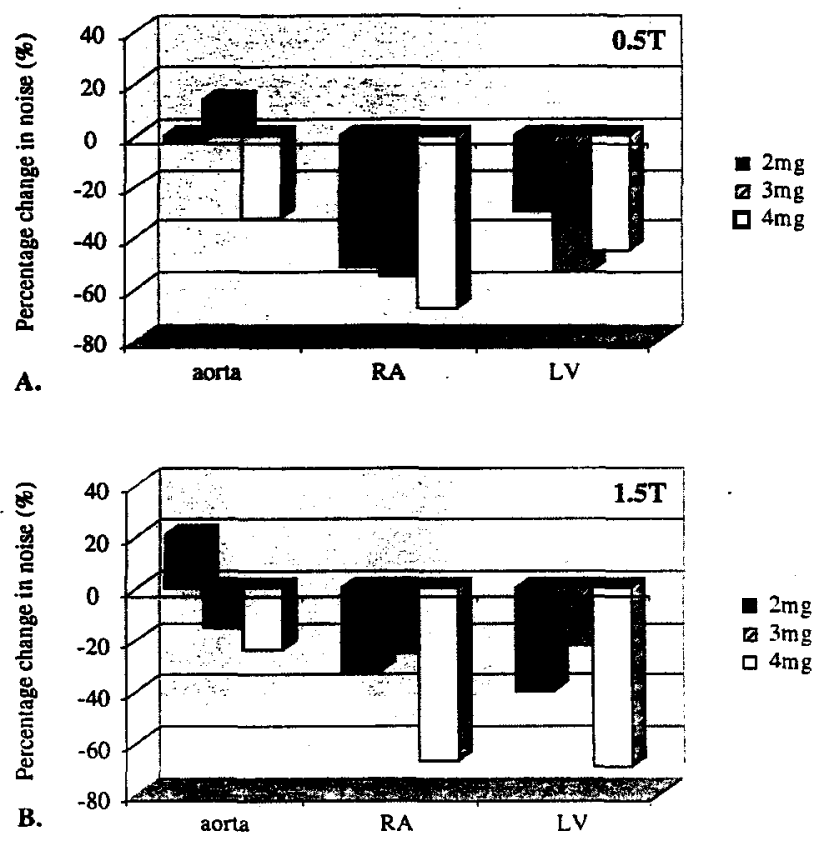

Figure 2. Percentage change in the blood flow artifact noise in the aorta, RA, and LV for each of the doses of NC100150 Injection (2, 3, and $4 \mathrm{mg} / \mathrm{kg}$ ). (A) 0.5-T data, (B) $1.5-\mathrm{T}$ data. At both 0.5 and $1.5 \mathrm{~T}$ there is a trend to a reduction in the noise in the aorta at the 4-mg/ $\mathrm{kg}$ dose. At $1.5 \mathrm{~T}$ there is a trend at all levels to a higher noise reduction with the $4-\mathrm{mg} / \mathrm{kg}$ dose. 
coxon signed-rank test. $p<0.05$ was regarded as significant.

\section{RESULTS}

There were no major adverse reactions to NC100150 Injection in any of 18 subjects after 14 days follow-up. There was no clinically significant change from baseline in the heart rate, systolic and diastolic blood pressures, oral temperature, respiration rate, or electrocardiograms (PR interval, the QRS duration, or the QT interval) during the study period at any of the three dose levels. No clinically significant changes were observed in the hematology and biochemistry parameters, the Ig and C levels, or the urinalysis.

\section{Spin-Echo Images}

There was a significant reduction in the blood pool flow artifact after the administration of NC100150 Injection at the level of the RA $(0.5 \mathrm{~T}, p<0.01 ; 1.5 \mathrm{~T}, p=$ $0.01)$ and $\operatorname{LV}(0.5 \mathrm{~T}, p=0.01 ; 1.5 \mathrm{~T}, p<0.01)$ but not at the level of the Ao ( $0.5 \mathrm{~T}, p=0.90 ; 1.5 \mathrm{~T}, p=0.90)$ (Table 1, Fig. 1). The effect was more marked at the highest dose; because of the small number of subjects at each dose, these differences were not statistically significant (Fig. 2).

The reduction in blood pool flow artifact by NC100150 Injection in the RA and LV was mirrored by a significant improvement of the subjective assessment of image quality at both field strengths (RA: $0.5 \mathrm{~T}, p=$ 0.015 and $1.5 \mathrm{~T}, p=0.011 ; \mathrm{LV}: 0.5 \mathrm{~T}, p=0.026$ and $1.5 \mathrm{~T}, p=0.26$ ). For the aorta there was a trend toward a subjective improvement in image quality at both field strengths. This probably reflects the reduction in blood pool artifact seen with the highest dose of NC100150 Injection (Fig. 2).

\section{Gradient-Echo Images}

Results at $0.5 \mathrm{~T}$

There was a small but insignificant increase in the LV blood pool SNR in the diastolic $(p=0.77)$ and systolic $(p=0.26)$ frames after the administration of NC100150 Injection [Table 2, Fig. 3(A)]. However, for the LV blood pool-myocardial SDNR there was a significant improvement in both diastolic $(p=0.01)$ and systolic $(p<$ 0.001 ) frames after the administration of NC100150 Injection (Table 2).

Interestingly, there was a reduction in the blood pool SNR with higher doses of NC100150 Injection [Fig. 4(A)]. Again, because of the small numbers involved, no significant difference in the SNR between doses was observed. However, the SDNR was maintained at the higher dose, despite the reduction in the SNR, because of a concordant reduction in the myocardial signal ( $48 \%$ at $4 \mathrm{mg}$ / $\mathrm{kg}$ ), which was not seen at the other doses (15\% increase at $2 \mathrm{mg} / \mathrm{kg}$, no change at $3 \mathrm{mg} / \mathrm{kg}$ ) [Fig. 4(B)]. Furthermore, with the higher doses of NC100150 Injection (3 and $4 \mathrm{mg} / \mathrm{kg}$ ), a rim of signal void was created, which was particularly helpful at defining the LV blood poolmyocardial border in the diastolic frames of these HLA cines (Fig. 5).

SI gradients across the LV blood pool-endocardial border showed an increase (i.e., better border definition) after the administration of $\mathrm{NC100150} \mathrm{Injection} \mathrm{in} \mathrm{both}$

Table 2

Gradient-Echo LV Blood Pool SNR Data and LV Blood Pool-Myocardial SDNR Data for All Doses of NC100150 Injection at Both Field Strengths

\begin{tabular}{|c|c|c|c|c|c|c|c|c|}
\hline & \multicolumn{4}{|c|}{$0.5 \mathrm{~T}$} & \multicolumn{4}{|c|}{$1.5 \mathrm{~T}$} \\
\hline & \multicolumn{2}{|c|}{ Diastole } & \multicolumn{2}{|c|}{ Systole } & \multicolumn{2}{|c|}{ Diastole } & \multicolumn{2}{|c|}{ Systole } \\
\hline & Pre & Post & Pre & Post & Pre & Post & Pre & Post \\
\hline SNR & $33 \pm 12$ & $34 \pm 11$ & $24 \pm 9$ & $27 \pm 6$ & $10 \pm 2$ & $18 \pm 2$ & $8 \pm 1$ & $16 \pm 4$ \\
\hline$t$-test & \multicolumn{2}{|c|}{0.77} & \multicolumn{2}{|c|}{0.26} & \multicolumn{2}{|c|}{$<0.001$} & \multicolumn{2}{|c|}{$<0.001$} \\
\hline$\%$ signal increase & \multicolumn{2}{|c|}{3} & \multicolumn{2}{|c|}{10} & \multicolumn{2}{|c|}{72} & \multicolumn{2}{|c|}{95} \\
\hline SDNR & $6 \pm 3$ & $9 \pm 4$ & $6 \pm 4$ & $15 \pm 3$ & $2 \pm 1$ & $5 \pm 1$ & $1 \pm 1$ & $8 \pm 3$ \\
\hline$t$-test & \multicolumn{2}{|c|}{0.01} & \multicolumn{2}{|c|}{$<0.001$} & \multicolumn{2}{|c|}{$<0.001$} & \multicolumn{2}{|c|}{$<0.001$} \\
\hline$\%$ signal increase & \multicolumn{2}{|c|}{56} & \multicolumn{2}{|c|}{141} & \multicolumn{2}{|c|}{221} & \multicolumn{2}{|c|}{916} \\
\hline
\end{tabular}

The $t$-test and \% signal increase compare data pre- and postcontrast for diastolic and systolic frames. 

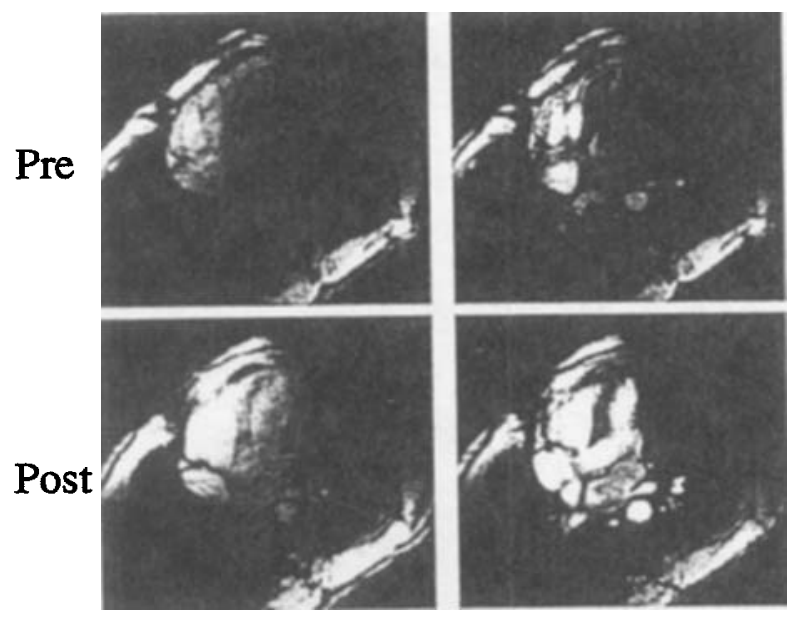

A.

Diastole

\section{Systole}
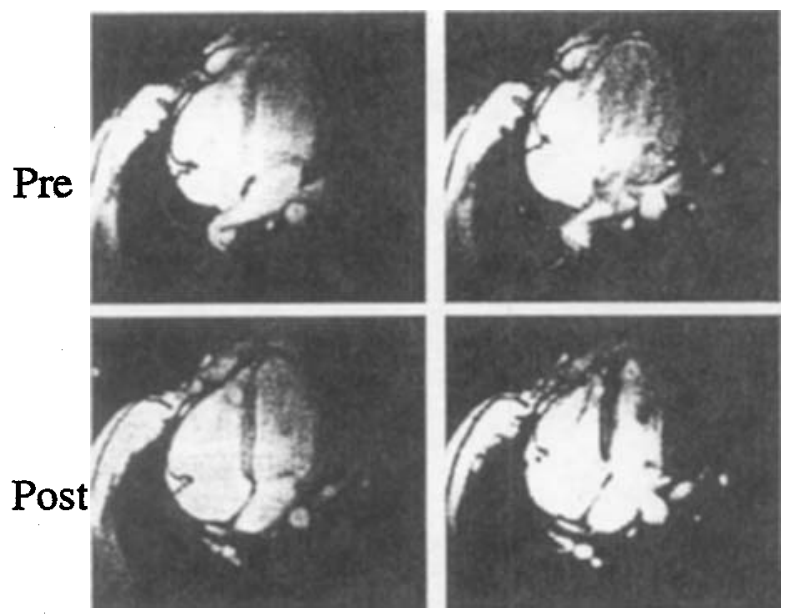

B.

Diastole

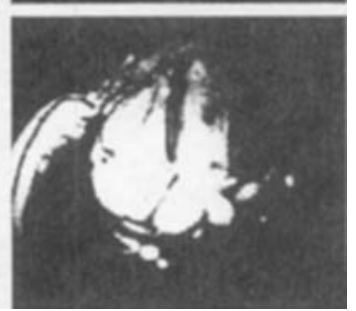

Systole

Figure 3. Horizontal long-axis gradient-echo images before and after the administration of NC100150 Injection. (A) Conventional gradient-echo images acquired at $0.5 \mathrm{~T}(2 \mathrm{mg} / \mathrm{kg})$. (B) Breathhold Turbo-FLASH images acquired at $1.5 \mathrm{~T}(3 \mathrm{mg} /$ $\mathrm{kg}$ ). The LV blood pool-myocardial border is clearly delineated in both systole and diastole after the administration of NC100150 Injection. However, the changes are best seen in the systolic frame at both field strengths.

the diastolic $(p<0.001)$ and systolic $(p<0.001)$ frames (Table 3). Abrupt changes in SI gradient were seen at the 3- and 4-mg/ $/ \mathrm{kg}$ doses related to the rim of signal void (Fig. 6), but no statistically significant difference in the
SI gradient increase was demonstrated between the given doses.

\section{Results at $1.5 \mathrm{~T}$}

There was a significant increase in both the LV blood pool SNR in the diastolic $(p<0.001)$ and systolic $(p<$ 0.001) frames [Table 2, Fig. 3(B)] and the LV blood pool-myocardial SDNR in the diastolic $(p<0.001)$ and systolic $(p<0.001)$ frames (Table 2$)$ after the administration of NC100150 Injection.

There was no significant difference between the given doses for the LV blood pool SNR and myocardial SNR [Fig. 4(A)]. There was a trend toward an increase in the LV blood pool-myocardium SDNR with increasing doses of NC100150 Injection [Fig. 4(C)]. However, because of the small numbers involved, these differences between doses remained statistically insignificant. No rim of signal void around the LV blood pool was seen at $1.5 \mathrm{~T}$ at any of the doses of $\mathrm{NC100150}$ Injection.

SI gradients across the LV blood pool-endocardial border showed an increase after the administration of NC100150 Injection in both the diastolic $(p=0.003)$ and systolic $(p=0.003$ ) frames (Table 3$)$. The abrupt changes in SI gradient seen at $0.5 \mathrm{~T}$, secondary to the rim of signal void, were not seen at $1.5 \mathrm{~T}$.

\section{Qualifative Results}

Qualitative score data for the overall HLA cine image quality correspond with the quantitative improvement in SDNR after NC100150 Injection $(0.5 \mathrm{~T}, p<0.01 ; 1.5$ $T, p<0.01$ ). Qualitative comparison of the wall definition after NC100150 Injection was also significant; lateral wall $(0.5 \mathrm{~T}, p<0.01 ; 1.5 \mathrm{~T}, p<0.01)$, apex $(0.5$ $\mathrm{T}, p<0.01 ; 1.5 \mathrm{~T}, p=0.01)$, and septum $(0.5 \mathrm{~T}, p<$ $0.01 ; 1.5 \mathrm{~T}, p=0.01$ ).

\section{DISCUSSION}

The intravascular contrast agent NC100150 Injection was given safely to 18 healthy volunteers. No major adverse reactions were seen, although events including dyspnea, abdominal pain, pruritus, headache, chest pain, injection site pain, and hypertension have been reported with other iron oxide-based contrast agents (11).

NC100150 Injection lowers blood T1 to below 100 msec for periods in excess of $2 \mathrm{hr}$ in humans and has an $\mathrm{R} 1$ relaxivity of $20 \mathrm{mM} / \mathrm{sec}$ at $0.5 \mathrm{~T}$ (Kessler et al., personal communication). It is thus well suited as a blood 

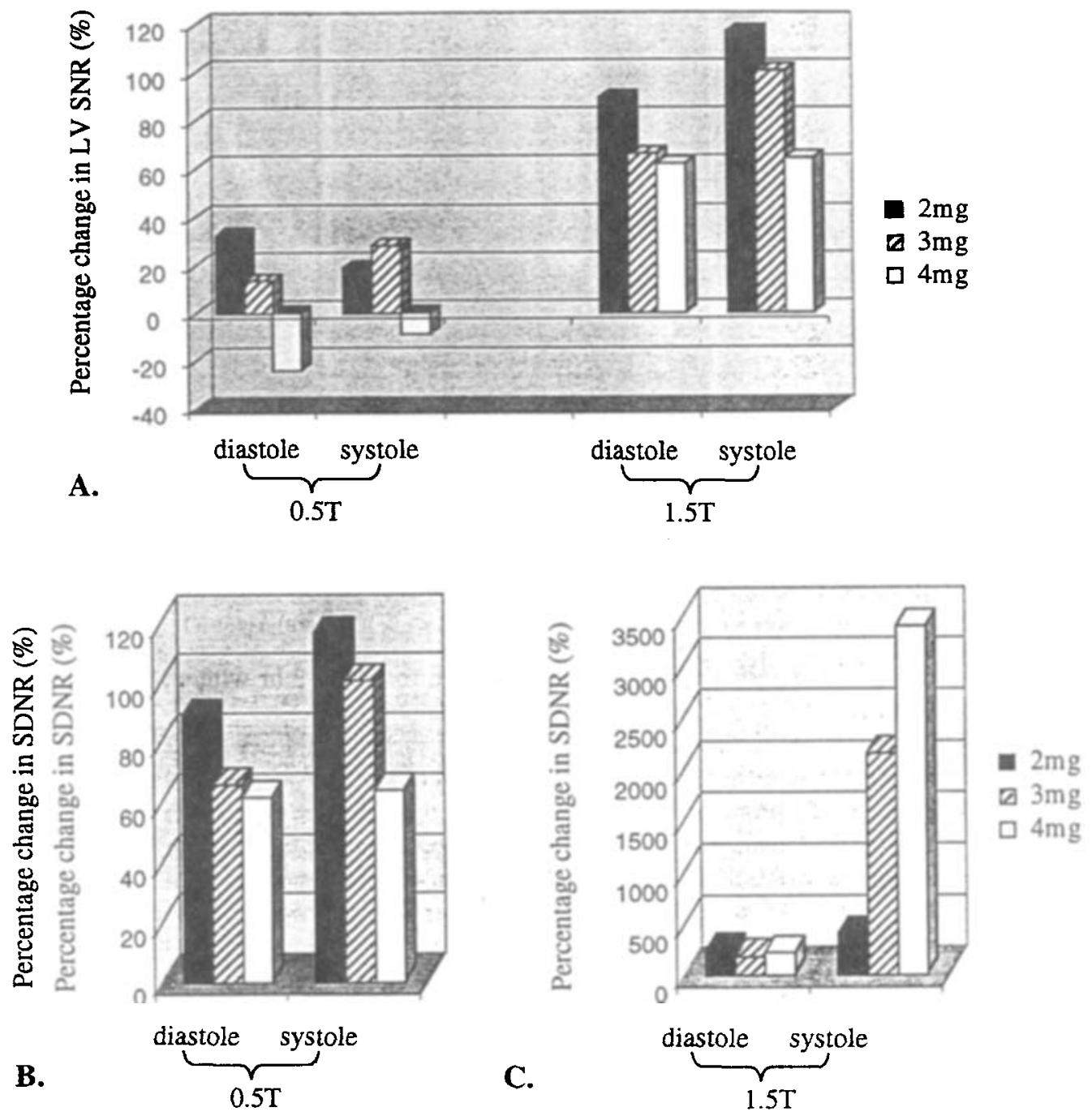

Figure 4. (A) Percentage change in the LV blood pool SNR in the diastolic and systolic frames at both field strengths for each of the doses of NC100150 Injection (2,3, and $4 \mathrm{mg} / \mathrm{kg}$ ). (B) Percentage change in the LV blood pool myocardial SDNR in the diastolic and systolic frames at $0.5 \mathrm{~T}$. (C) Percentage change in the LV blood pool myocardial SDNR in the diastolic and systolic frames at $1.5 \mathrm{~T}$.

pool contrast agent. With the Turbo-FLASH sequence (short TE and TR) used at $1.5 \mathrm{~T}$, the $\mathrm{T} 1$ effects of NC100150 Injection dominate the images, leading to an improvement in both the SNR and the SDNR after the administration of contrast. Furthermore, because blood pool saturation is reduced by NC100150 Injection, a high flip angle was used in the postcontrast sequence, reducing the myocardial signal and further enhancing the SDNR. The increase in the diastolic frame SDNR seen in this study was nearly twice that seen in the preliminary study of Stillman et al. (12), using Ferumoxtran (Advanced Magnetic Inc., Cambridge, MA) (221 versus $128 \%$, ratio 1.7) at $1.5 \mathrm{~T}$, although it must be noted that the sequences differed in the two studies with a shorter TE and T1weighting used in our study. There was a trend toward an increase in the SDNR with higher NC100150 Injection doses, related to the increase in $\mathrm{T} 1$ recovery.

In the conventional gradient-echo images at $0.5 \mathrm{~T}$, 


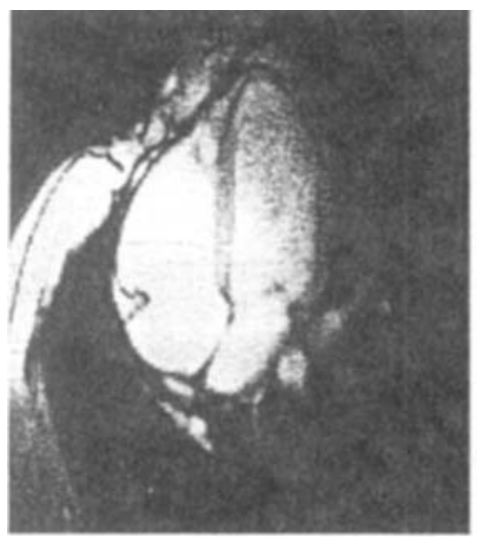

$2 \mathrm{mg}$

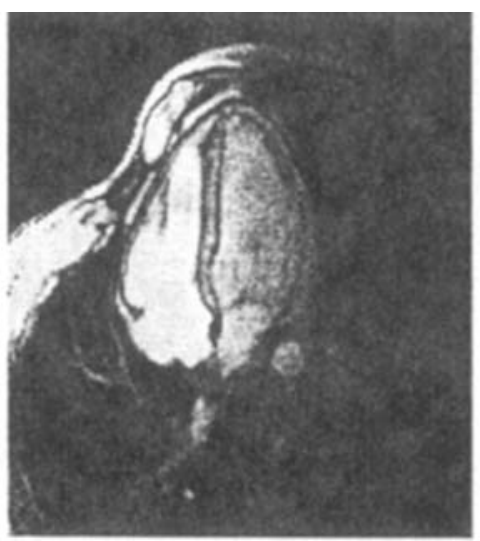

$3 \mathrm{mg}$

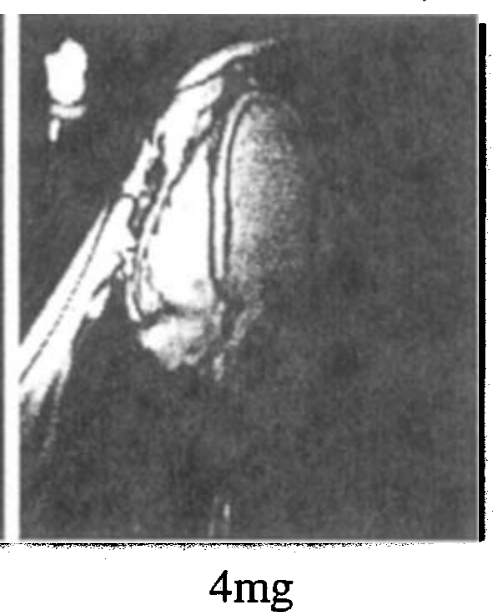

Figure 5. Horizontal long-axis gradient-echo images at $0.5 \mathrm{~T}$ after the administration of NC100150 Injection. At the 3- and 4$\mathrm{mg} / \mathrm{kg}$ doses, a rim of signal void can clearly be seen delineating the LV blood pool-myocardial border.

there was no significant increase in the SNR because of the long TE ( $8 \mathrm{msec}$ ) used, causing the T1 and T2* effects to balance each other. However, with the high flip angle used in the postcontrast imaging sequence, myocardial signal was reduced, whereas the $T 1$ properties of the contrast agent maintain the LV blood pool signal, improving the LV blood pool-myocardial SDNR. The SDNR increase for the diastolic (56 versus $26 \%$, ratio 2.2 ) and systolic (141 versus $67 \%$, ratio 2.1 ) frames was over twice that seen in the study of Pennell et al. (13), using gadopentetate dimeglumine (Magnevist, Berlex, Wayne, NJ) at $0.5 \mathrm{~T}$. These differences can be accounted for by the lack of myocardial enhancement by the intravascular contrast agent. Furthermore, the improvement in contrast with gadopentetate dimeglumine is limited to $20 \mathrm{~min}$, whereas with NC100150 Injection, imaging can continue for up to $2 \mathrm{hr}$ without reduction in blood pool enhancement.

With increasing doses of NC100150 Injection at 0.5 $\mathrm{T}$, a rim of signal void was visualized, which further enhanced the observer's ability to distinguish between the LV blood pool and the myocardium. This signal void is secondary to the cancellation of opposing phase spins in the contrast-enhanced blood pool and the myocardium, occurring in the same pixel. The effect depends on the balance between the $\mathrm{T} 1$ and $\mathrm{T} 2 *$ effects of the agent, the dose given, and the sequence TE. Thus, if a sequence TE of $2 \mathrm{msec}$ had been used, the rim signal void would not have been seen at any of the three doses, whereas if a $\mathrm{TE}$ of $12 \mathrm{msec}$ had been used, the rim of signal void would have been present in at all doses used.

Automated LV contour algorithms search for maxi-

Table 3

Midseptum LV Blood Pool-Endocardial SI Gradient Data for All Doses of NC100150 Injection at Both Field Strengths

\begin{tabular}{|c|c|c|c|c|c|c|c|c|}
\hline & \multicolumn{4}{|c|}{$0.5 \mathrm{~T}$} & \multicolumn{4}{|c|}{$1.5 \mathrm{~T}$} \\
\hline & \multicolumn{2}{|c|}{ Diastole } & \multicolumn{2}{|c|}{ Systole } & \multicolumn{2}{|c|}{ Diastole } & \multicolumn{2}{|c|}{ Systole } \\
\hline & Pre & Post & Pre & Post & Pre & Post & Pre & Post \\
\hline SI gradient & $1.5 \pm 0.9$ & $6.0 \pm 3.0$ & $1.6 \pm 0.9$ & $5.5 \pm 2.4$ & $0.7 \pm 0.3$ & $2.1 \pm 0.9$ & $0.8 \pm 0.4$ & $2.9 \pm 1.6$ \\
\hline$t$-test & \multicolumn{2}{|c|}{$<0.001$} & \multicolumn{2}{|c|}{$<0.001$} & \multicolumn{2}{|c|}{0.003} & \multicolumn{2}{|c|}{0.003} \\
\hline$\%$ SI increase & \multicolumn{2}{|c|}{291} & \multicolumn{2}{|c|}{242} & \multicolumn{2}{|c|}{184} & \multicolumn{2}{|c|}{276} \\
\hline
\end{tabular}

The $t$-test and \% SI increase compare data pre- and postcontrast for diastolic and systolic frames. 


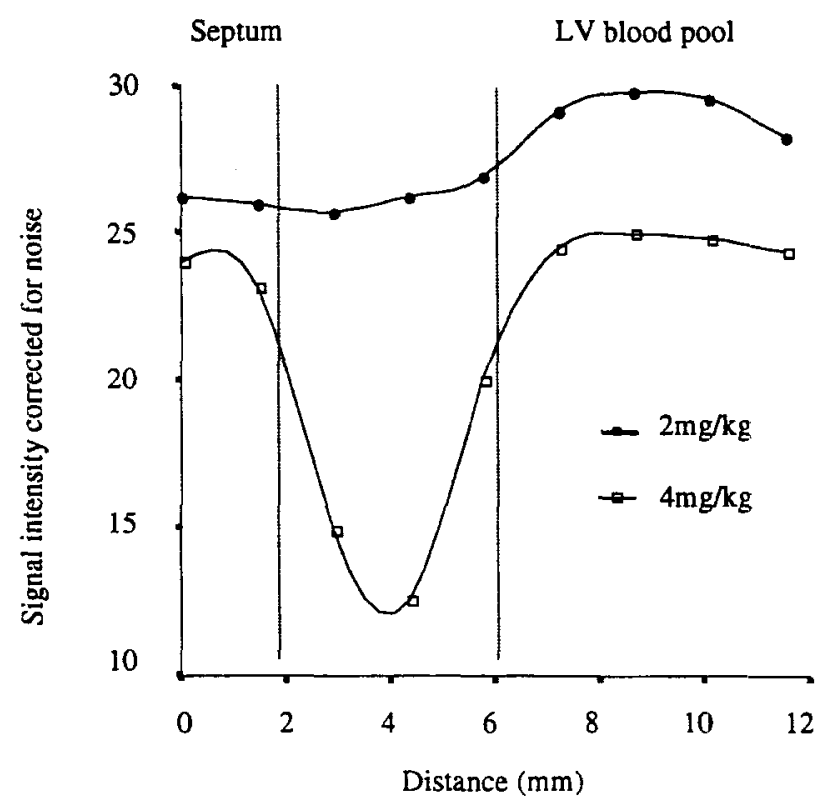

Figure 6. Plots of SI image profiles (corrected for background noise) across the midseptum for diastolic images at $0.5 \mathrm{~T}$ (2 and $4 \mathrm{mg} / \mathrm{kg}$ ). The reduction in LV blood pool signal and myocardial signal are seen at the higher dose. However, the SI gradient is greater at $4 \mathrm{mg} / \mathrm{kg}$, secondary to the rim of signal void, which is represented by the signal dip seen in the $4-\mathrm{mg} / \mathrm{kg}$ SI image profile.

mum intensity gradients at the endocardial and epicardial borders (14). The significant improvement in the SI gradients across the LV blood pool-endocardial border after the administration of NC100150 Injection should therefore improve automated segmentation capabilities.

The spin-echo sequence uses a long TE and TR. T2* effects dominate, leading to a reduction in the blood pool flow signal artifact and an improvement in the image quality. As would be expected, there was a definite trend toward an increase in this effect with increasing dose of NC100150 Injection, although this remained insignificant because of the small number of subjects studied.

\section{Study Limitations}

In general, it is good practice when studying contrast agents to compare the same sequence before and after contrast. However, it was believed that the optimal sequence parameters should be used for both the pre- and postcontrast images. Thus, we used our currently optimized clinical sequence parameters for precontrast imaging and a modified sequence (high flip angle) after con- trast. This postcontrast sequence had been previously optimized during phantom studies and was used to enhance the T1-weighting of the image. If this high flip angle sequence had been used before contrast, very little signal would have been generated, and the results would have been further enhanced.

\section{CONCLUSIONS}

The intravascular iron oxide particle contrast agent, NC100150 Injection, can be given safely to humans at doses of between 2 and $4 \mathrm{mg} / \mathrm{kg}$. At these doses, there is an improvement in both qualitative and quantitative image parameters in conventional spin-echo images at 0.5 and $1.5 \mathrm{~T}$, conventional gradient-echo images at 0.5 $\mathrm{T}$ and breathhold, and Turbo-FLASH gradient-echo images at $1.5 \mathrm{~T}$.

Further studies are now required to identify the lowest administration dose, optimize pulse sequences, and establish whether automated edge detection algorithms will be improved in the presence of NC100150 Injection in the blood pool.

\section{ACKNOWLEDGMENTS}

This work was supported by a grant from Nycomed Imaging AS. A.M.T., J.R.P, J.K., P.D.G., P.J., and D.N.F. are supported by the Coronary Disease Research Association. G.Z.Y is supported by the Wellcome Trust.

\section{REFERENCES}

1. Prince MR, Grist TM and Debatin JF. $3 D$ Contrast $M R$ Angiography. Berlin: Springer Verlag; 1997.

2. Wilke $\mathrm{N}$, Kroll $\mathrm{K}$, Merkle $\mathrm{H}$, Wang $\mathrm{Y}$, Ishibashi $\mathrm{Y} ; \mathrm{Xu}$ $X$, Zhang J, Jerosch-Herold $M$, Müller A, Stillman AE, Bassingthwaighte JB, Bache R and Ugurbil K. Regional myocardial blood volume and Flow: First pass imaging with polylysine-Gd-DTPA. J Magn Reson Imaging, 1995; 5:227-237.

3. Dulce MC, Mostbeck GH, Friese KK. Caputo GR and Higgins CB. Quantification of the left ventricular volumes and function with cine MR imaging: Comparison of geometric models with three-dimensional data. Radiology, 1993; 188:371-376.

4. Sakuma H, Fujita N, Foo TK, Caputo GR, Nelson SJ, Hartiala J, Shimakawa A and Higgins CB. Evaluation of left ventricular volume and mass with breath-hold cine MR imaging. Radiology, 1993; 188:377-380.

5. Bogaert JG, Bosmans HT, Rademakers FE, Bellon EP, Herregods MC, Verschakelen JA, Van de Werf F and 
Marchal GJ. Left ventricular quantification with breathhold MR imaging: Comparison with echocardiography. MAGMA, 1995; 3:5-12.

6. Wiesmann F, Gatehouse PD, Panting JR, Taylor AM, Firmin DN and Pennell DJ. Comparison of fast spiral, echo planar and FLASH magnetic resonance imaging for cardiac volumetry at $0.5 \mathrm{~T}$. $J$ Magn Reson Imaging (in press).

7. Fleagle SR, Thedens DR, Stanford W, Pettigrew RI, Reichek $\mathrm{N}$ and Skorton DJ. Multicenter trial of automated border detection in cardiac MR imaging. J Magn Reson lmaging, 1993; 3:409-415.

8. Baldy C, Doueck P, Croisille P, Magnin IE, Revel D and Amiel $M$. Automated myocardial edge detection from breath-hold cine-MR images: Evaluation of left ventricular volumes and mass. Magn Reson Imaging, 1994; 12 : 589-598.

9. Mitchell DG. MR imaging contrast agents-What's in a name? J Magn Reson Imaging, 1997; 7:1-4.
10. Wolfe SD and Balaban RS. Assessing contrast on MR images. Radiology, 1996; 202:25-29.

11. Petersein J, Saini S and Weissleder R. Liver II: Iron oxide-based reticuloendothelial contrast agents for MR imaging. Clinical review. Magn Reson Imag Clin North Am, 1996; 4:53-60.

12. Stillman $\mathbf{A E}$, Wilke $\mathbf{N}$ and Jerosch-Herold $\mathrm{M}$. Use of an intravascular $\mathrm{T} 1$ contrast agent to improve MR cine myocardial-blood pool definition in man. J Magn Reson Imaging, 1997; 7:765-767.

13. Pennell DJ, Underwood SR and Longmore DB. Improved cine MR imaging of left ventricular wall motion with gadopentetate dimeglumine. J Magn Reson Imaging, 1993; 3:13-19.

14. Wang JZ, Tumer DA and Chatuape MD. Fast, interactive algorithm for segmentation of a series of related images: Application of volumetric analysis of MR images of the heart. J Magn Reson Imaging, 1992; 2:575582. 